\title{
Effects of Long-Term Developmental Patterns of Adiposity on Levels of C-Reactive Protein and Fibrinogen among North-American Men and Women: The Spokane Heart Study
}

\author{
Trynke Hoekstra $^{\text {a, b }} \quad$ Celestina Barbosa-Leiker ${ }^{d} \quad$ Bruce R. Wright $^{c}$ \\ Jos W.R. Twisk ${ }^{b}$ \\ ${ }^{a}$ Faculty of Earth and Life Sciences, Department of Health Sciences and the EMGO Institute \\ of Health and Care Research, VU University, ${ }^{\mathrm{b}}$ Department of Epidemiology and Biostatistics \\ and the EMGO Institute of Health and Care Research, VU University Medical Center, \\ Amsterdam, The Netherlands, ' ${ }^{2}$ Health and Wellness Services, Washington State University \\ Pullman, WA, ${ }^{d}$ College of Nursing, Washington State University, Spokane, WA, USA
}

\begin{abstract}
This study examined the heterogeneity in BMI development by identifying distinct developmental trajectories. These trajectories were further investigated by relating them to markers of lowgrade inflammation later in life. Data from approximately 400 healthy volunteers participating in the Spokane Heart Study were collected in 2-year intervals, and four waves of data were available for the current analyses. Body weight was measured by BMI and low-grade inflammation by high-sensitivity C-reactive protein (CRP) and fibrinogen. Up to date statistical techniques, i.e. latent class growth models, were used to analyse heterogeneity in body weight, and linear regressions were run to analyse possible associations between trajectories of body weight and CRP/fibrinogen levels. Six trajectories were identified (three stable, two increasing, and one decreasing) which differed significantly on CRP/fibrinogen levels, highlighting the importance of weight trajectories. The differences were only partly explained by variations in lifestyle habits.
\end{abstract}

\section{Introduction}

Worldwide, the prevalence of being overweight (i.e., a BMI of $25-29.9 \mathrm{~kg} / \mathrm{m}^{2}$ ) or obese $\left(\mathrm{BMI} \geq 30 \mathrm{~kg} / \mathrm{m}^{2}\right)$ is rapidly increasing across all age, gender, and racial groups [1]. Ample studies have shown that being overweight or obese is associated with many chronic diseases such as arthritis [2], certain cancers [3], and sleep apnoea [4]. Further, it has been shown that these adiposity categories are associated with cardiovascular diseases (CVD). High levels of 
Hoekstra et al.: Effects of Long-Term Developmental Patterns of Adiposity on Levels of C-Reactive Protein and Fibrinogen among North-American Men and Women: The Spokane Heart Study

BMI have consistently been found to be strong risk factors, amongst others, for hypertension [5] and stroke [6]. However, markers of low-grade inflammation might in part explain these relationships $[7,8]$. Well-known examples of such markers include C-reactive protein (CRP) and fibrinogen.

CRP is a protein produced in the liver in response to inflammation or due to increased adipocyte activity $[9,10]$. The protein has been extensively investigated, especially by crosssectional studies, and CRP has been shown to be elevated in obese individuals from various study populations [9-11]. Moreover, CRP has also been found to be an independent marker of elevated CVD risk $[9,12]$. Fibrinogen, which is also produced in the liver, is a marker of both inflammation and haemostasis and is known to predict future CVD as well [13]. Studies investigating fibrinogen and adiposity demonstrated elevated fibrinogen values in overweight and obese individuals [14].

Many longitudinal studies have shown that weight gain $[15,16]$ and greater weight variability over time $[17,18]$ are associated with an increase in levels of several low-grade inflammation markers, whereas others did not find any association at all [19]. These inconsistent findings are one of the reasons why weight variability research remains of high interest; in addition this is partly driven by the rapid progress that has been made in the statistical analysis of longitudinal data. Up to date techniques, i.e., latent class growth models (LCGM), make possible the analysis of distinct BMI trajectories and of subsequent associations with markers of low-grade inflammation. It has recently been shown [20-22] that the development of BMI is heterogeneous over time, where not only weight loss or weight gain in itself but also distinct trajectories of BMI may be of importance for the quantification of various health risks. For example, Mustillo et al. [23] identified four distinct obesity trajectories in a cohort of 9- to 16-year-old children, each with distinct consequences for psychopathology. Further, Kuchibhatla et al. [24] identified three distinct trajectories of BMI in a cohort of elderly participants, whereas Botoseneanu and Liang [25] identified five distinct trajectories in a cohort of subjects of similar age. In all studies, distinct trajectories of BMI coincided with differential health or disease consequences. Given the increasing obesity epidemic, investigating the subsequent health and disease consequences of distinct BMI trajectories in a range of cohorts is critical. Particularly the possibility to identify high-risk individuals who may need supplementary treatment or preventive measures in addition to the standard care or prevention is important. In this light, the evaluation of (health and disease consequences of) distinct trajectories might also allow for the identification of low-risk individuals who need less treatment. Using 'conventional' statistical approaches (e.g., calculating BMI change scores) would probably mask this heterogeneity in development.

The aim of the current study was twofold: First, we analysed the heterogeneity of BMI development over time, thereby determining the number and characteristics of distinct developmental patterns of BMI in an adult study population. Secondly, we related these distinct subgroups to late-life CRP- and fibrinogen levels, revealing subgroups specifically at risk for future CVD.

\section{Material and Methods}

\section{The Spokane Heart Study}

The Spokane Heart Study is a longitudinal study of healthy local participants investigating the development of pre-clinical atherosclerosis in order to predict the risk for coronary artery disease from traditional and non-traditional factors [26-29]. Beginning in 1994, participants were enrolled every 2 years until 2006, and data was collected at 2-year intervals. Visits therefore took place in different years for different participants, and not all participants were followed up an equal number of times. In total, 1,147 participants in the Spokane Heart Study were visited at least once. 
Hoekstra et al.: Effects of Long-Term Developmental Patterns of Adiposity on Levels of C-Reactive Protein and Fibrinogen among North-American Men and Women: The Spokane Heart Study

The study was reviewed and approved by the institutional review board of the participating university and community hospitals, and written informed consent was given by all participants at all measurement occasions. For consistency in sample sizes over time and to avoid possible period effects, only visits 1-4 were analysed. Participants who had multiple (three or more) measurements [22] of body weight over time $(n=441)$ and from whom valid blood samples were available $(n=407)$ were included. The characteristics of this selection were compared to the 1,147 participants of the Spokane Heart Study and, at the first visit, showed no relevant differences on BMI, CRP or fibrinogen (BMI: $26.66 \pm 4.42 \mathrm{~kg} / \mathrm{m}^{2}$ vs. $27.24 \pm$ $4.39 \mathrm{~kg} / \mathrm{m}^{2}$; CRP: $2.5 \pm 3.80 \mathrm{mg} / \mathrm{dl}$ vs. $2.87 \pm 3.60 \mathrm{mg} / \mathrm{dl}$; fibrinogen: $319.73 \pm 207.29 \mathrm{mg} / \mathrm{dl}$ vs. $321.78 \pm$ $124.24 \mathrm{mg} / \mathrm{dl})$.

In total, 203 (49.9\%) women and 204 men were included in the study. Participants in the Spokane Heart Study were predominately Caucasian $(>98 \%)$, and the obesity rates at visit $1(16.2 \%)$ conducted around 1994 for this sample were comparable to the nationwide obesity rates (14.4\%) [30].

Adiposity

Adiposity was quantified by means of the BMI. The BMI was calculated by dividing body mass (kg) by height squared (meters). Body mass was measured using a calibrated scale, and height was measured using a wall-mounted stadiometer, generally available at community hospitals throughout the USA.

\section{C-Reactive Protein and Fibrinogen}

Fasting blood samples were collected in the morning of visit 1 as well as visit 4 and separated into serum and plasma by centrifugation. High-sensitivity CRP in plasma was analysed using nephelometric assays at the Center for Disease Control and Prevention (CDC), Atlanta, GA, USA. As recommended by the CDC and the American Heart Association, levels $>10 \mathrm{mg} / \mathrm{dl}$ were not included in the analyses because they may indicate active infection or trauma [31]. Fibrinogen was analysed by the Pathology Associates Medical Laboratory, a nationally certified laboratory in Spokane, WA, and also expressed in $\mathrm{mg} / \mathrm{dl}$.

\section{Covariates}

Baseline lifestyle covariates were utilised. Smoking status was asked in a questionnaire, and it was stated whether the participant was a smoker or a non-smoker. Physical exercise levels were determined by a self-report questionnaire measuring the extent of engagement in several sports activities. The answers to all questions were combined to form a binary variable indicating whether or not a participant engaged regularly (approximately $5 \mathrm{~h} /$ week, including all levels of intensity according to the 2008 physical activity guidelines for adults developed by the CDC control) in sportive activities. Dietary information was determined by a self-report questionnaire asking whether the participant was on a restricted diet (i.e., low-fat and/or low-salt and/or low-cholesterol and/or vegetarian); accordingly, a binary variable (restricted diet yes/no) was included in the analyses. Information on current oral contraceptive usage (females only) was obtained by a self-report questionnaire and scored as a binary variable (users versus non-users). Information on usage of other relevant medication known to influence low-grade inflammation (i.e., blood pressure medication, cholesterol medication, and diabetes medication) was obtained from a self-report questionnaire. This variable was also binary coded and included in the analyses as such.

Possible effect modification by gender was assessed by creating interaction terms (class membership dummies $\times$ gender) and evaluating their $\mathrm{p}$ values.

\section{Statistical Methods}

Analyses were conducted using the Mplus $7.0[32,33]$ and the PASW 20.0 software packages.

Analyses were conducted in two steps. All continuous outcome variables were checked for normality and log transformed if necessary, and all assumptions underlying the analyses were checked. Significance results were given as two-sided $p$ values unless stated otherwise, where a $p$ value $<0.05$ was regarded as statistically significant.

\section{Step 1 - Latent Class Growth Modelling}

In step 1, distinct developmental patterns of BMI were identified by means of LCGM [34-37]. LCGM is an up to date statistical technique in obesity research. The aim of the technique is to capture the heterogeneity of BMI development over time in k numbers of subgroups (or classes) with comparable BMI development. Each subgroup has its own growth parameters (intercept, slope) which are unobserved (or latent) continuous variables. 
Hoekstra et al.: Effects of Long-Term Developmental Patterns of Adiposity on Levels of C-Reactive Protein and Fibrinogen among North-American Men and Women: The Spokane Heart Study

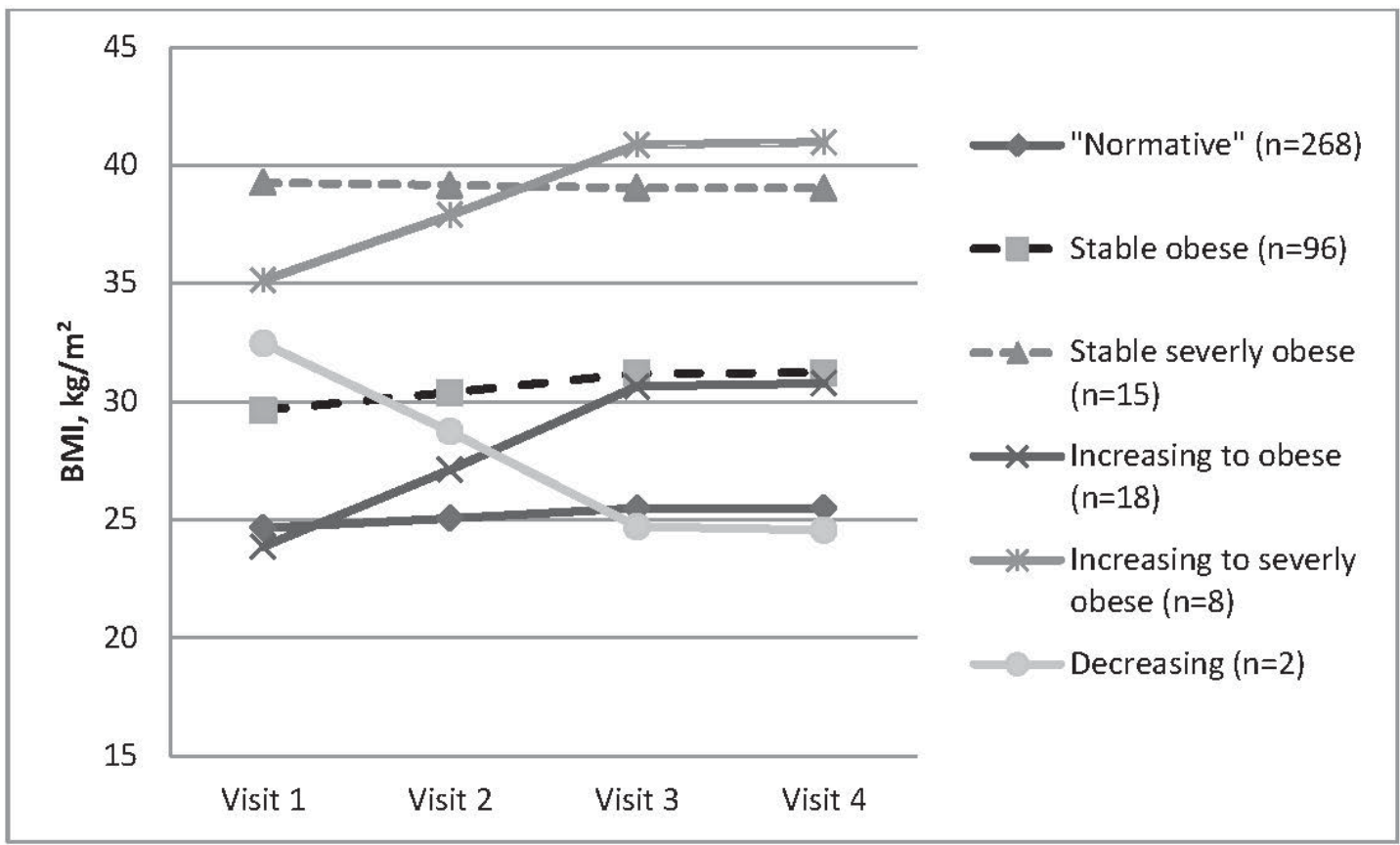

Fig. 1. Six developmental trajectories of BMI.

\section{Class Identification}

To determine the optimal number of latent classes, a forward procedure [22, 37] was performed, starting with a 1-class solution (i.e., there were no subgroups in the study sample; all individuals followed the same trajectory over time), then adding more classes one at a time to investigate whether or not the model fit improved due to the additional class. We used several model fit indices and other criteria to compare the fit between subsequent models, as suggested in the literature [38]. First, we used two model fit indices: the Bayesian information criterion (BIC) and the bootstrap likelihood ratio test (BLRT). The BIC is commonly used within latent variable modelling analyses, considering both the likelihood of the model as well as the number of parameters in the model; a lower BIC value shows a better model fit (a decrease of at least 10 points is suggested as sufficient improvement) [39]. The BLRT [40] provides a p value, indicating that a model with one less class ( $\mathrm{k}-1$-class model) has to be rejected in favour of the k class model. Both fit indices have proven to be consistent indicators of the optimal number of classes [38]. To further determine the optimal number of classes, we secondly looked at the posterior probabilities of belonging to each of the $\mathrm{k}$ classes [38]. Based on these probabilities, study participants were assigned to their most likely class, and only solutions with posterior probabilities of $0.8-0.9$ or higher were accepted $[37,38]$. The probability for the class the individual was assigned to should be considerably higher than the other probability/probabilities. In this way, the classes clearly differed from each other. Additionally, we considered the usefulness of the separate classes in practice. This was done in two ways: by examining the shape of the trajectories and by the number of individuals in each class. Clinically uninterpretable solutions were rejected in favour of interpretable solutions [37].

\section{Step 2 - Linear Regression Analyses}

The first step of the analyses provided us with a classification of the study population into distinct subgroups, based on the development of BMI. The classification was coded as a categorical variable with $\mathrm{k}$ numbers of categories or classes. To study the possible association between the distinct subgroups and CRP as well as fibrinogen, several linear regression analyses were performed with the markers of lowgrade inflammation as outcome variables. First, 'crude' analyses were conducted in which class membership was related to CRP or fibrinogen, correcting only for age, gender, relevant medication use and oral contraceptive usage. Secondly, adjusted analyses were carried out, adding the lifestyle covariates to the regression model. 
Hoekstra et al.: Effects of Long-Term Developmental Patterns of Adiposity on Levels of C-Reactive Protein and Fibrinogen among North-American Men and Women: The Spokane Heart Study

Table 1. Model fit indices

\begin{tabular}{lllll}
\hline $\begin{array}{l}\text { Number of } \\
\text { classes }\end{array}$ & $\begin{array}{l}\text { Bayesian } \\
\text { information } \\
\text { criterion }\end{array}$ & $\begin{array}{l}\text { Bootstrapped } \\
\text { likelihood ratio test }\end{array}$ & $\begin{array}{l}\text { Average posterior } \\
\text { probability (min-max) }\end{array}$ & $\begin{array}{l}\text { Number of subjects } \\
\text { per class }\end{array}$ \\
\hline 1 & $7,604.838$ & not available & 1.00 & 407 \\
2 & $7,561.913$ & $<0.001$ & $0.916(0.843-0.988)$ & $385 / 22$ \\
3 & $7,532.887$ & $<0.001$ & $0.933(0.873-0.986)$ & $8 / 377 / 22$ \\
4 & $7,524.385$ & $<0.001$ & $0.922(0.824-1.00)$ & $15 / 2 / 27 / 363$ \\
5 & $7,521.011$ & $<0.001$ & $0.897(0.821-1.00)$ & $2 / 13 / 16 / 364 / 12$ \\
6 & $7,527.809$ & 0.08 & $0.869(0.790-1.00)$ & $2 / 15 / 268 / 8 / 18 / 96$ \\
\hline
\end{tabular}

Results are were reported as unstandardised regression coefficients, indicating the mean difference in the markers of low-grade inflammation between the distinct trajectories of BMI. Corresponding $95 \%$ confidence intervals and two-sided $p$ values are presented alongside.

The $\mathrm{p}$ values of the interaction terms investigating effect modification by gender were all non-significant ( $p=0.05,0.429,0.616,0.649$ and 0.670$)$, and therefore no stratification by gender was made.

Advantages of this Approach

LCGMs allow for the empirical identification and subsequent analysis of otherwise invisible distinct subgroups of individuals with similar BMI trajectories over time (see above).

Additional Analyses

In order to determine if 'conventional' statistical approaches provided any further information and/or stronger, or weaker prediction of CRP and fibrinogen by BMI, we calculated change scores of BMI (BMI at visit 4 minus BMI at visit 1) and related this continuous variable to CRP and fibrinogen, using the regression analysis steps described above in step 2 .

\section{Results}

The 'best' model of the LCGM is visualised in figure 1. Based on the model fit indices and additional information available (see table 1), selecting the 'best' model was relatively difficult because of some inconsistency in model fit indices. Based on the BIC, a 5-class solution could be considered as being the 'best' model, but the BLRT pointed towards a 6-class model. After taking into account the clinical interpretation of the 4-, 5- and 6-class models in particular, a final decision for the 6-class model was made, also because the BLRT had proven to be a very consistent model fit index [38]. Therefore, the best LCGM solution was a 6-class model, where most participants could be classified in a 'normative' class which is characterised by stable BMI values around $25 \mathrm{~kg} / \mathrm{m}^{2}$. Subsequently, two more stable subgroups emerged: a stable obese $(n=96)$ and a stable severely obese $(n=15)$ subgroup. The stable obese subgroup was characterised by stable BMI values around $30 \mathrm{~kg} / \mathrm{m}^{2}$, although slight increases were apparent after visit 2 . The stable severely obese subgroup was characterised by stable BMI values of just under $40 \mathrm{~kg} / \mathrm{m}^{2}$. Further, two groups commenced with a BMI increase which was then levelled off, starting at different baseline values (normal weight $(n=18)$ and obese $(n=8)$ ). The first subgroup showed a steep increase between visit 1 and 3 (approximately 7 BMI points); an increase of similar extent was visible for the latter.

Finally, a small group $(\mathrm{n}=2)$ showed a decrease from an obese baseline state (BMI values between 30 and $35 \mathrm{~kg} / \mathrm{m}^{2}$ ) towards a 'normative' state (BMI values similar to the normative subgroup). Because of the small sample size, this class was not analysed further. The fit infor- 
Hoekstra et al.: Effects of Long-Term Developmental Patterns of Adiposity on Levels of C-Reactive Protein and Fibrinogen among North-American Men and Women: The Spokane Heart Study
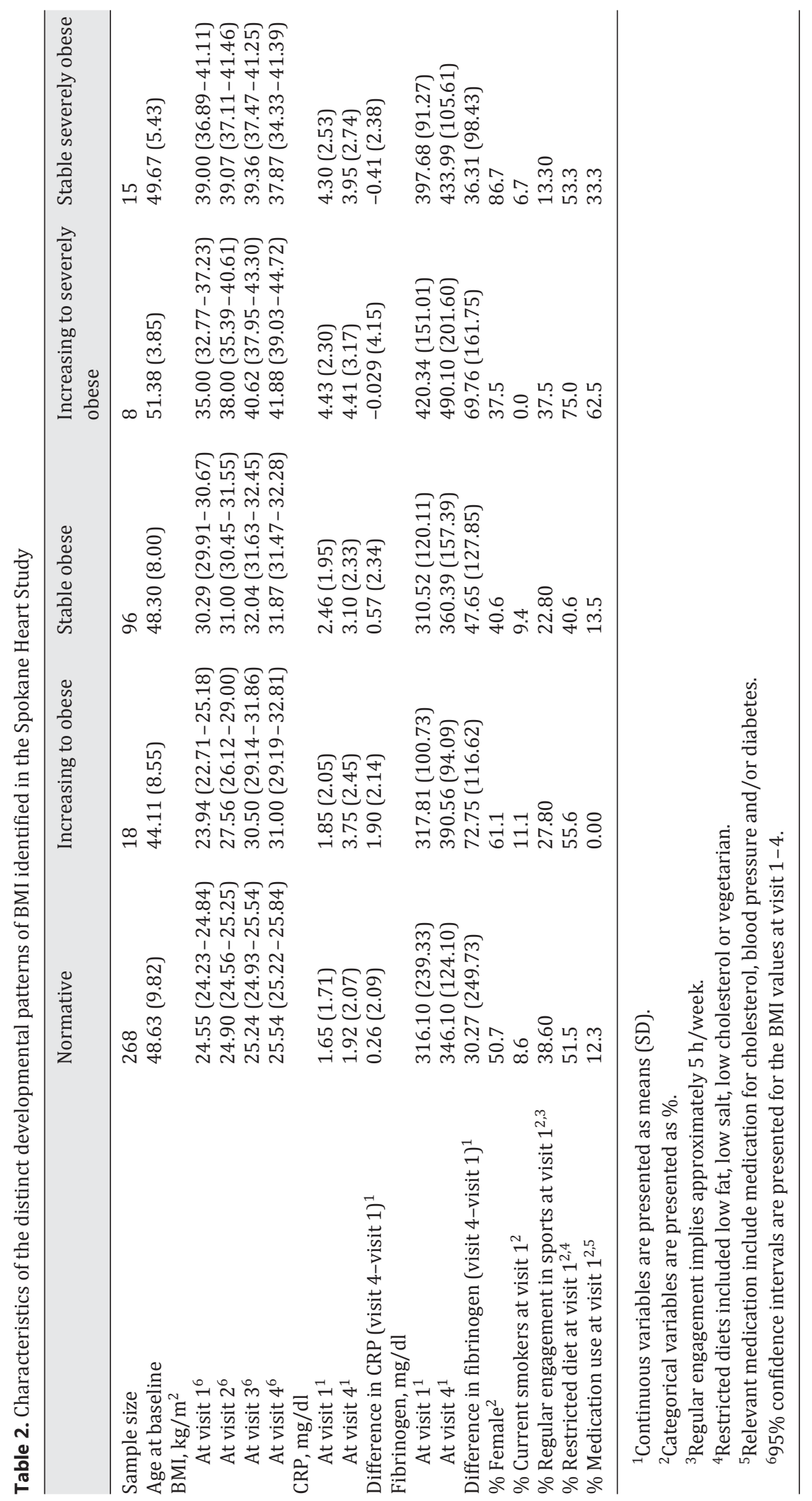
Hoekstra et al.: Effects of Long-Term Developmental Patterns of Adiposity on Levels of C-Reactive Protein and Fibrinogen among North-American Men and Women: The Spokane Heart Study

Table 3. Unstandardised regression coefficients (b), $95 \%$ confidence intervals and $p$ values for the associations between BMI-trajectory membership and CRP

\begin{tabular}{|c|c|c|c|c|c|c|}
\hline & \multicolumn{3}{|c|}{ Model $1^{\mathrm{a}}$} & \multicolumn{3}{|c|}{ Model $2^{\mathrm{b}}$} \\
\hline & $b^{d}$ & $\begin{array}{l}95 \% \text { confidence } \\
\text { interval }\end{array}$ & $\mathrm{p}$ value & $b^{d}$ & $\begin{array}{l}95 \% \text { confidence } \\
\text { interval }\end{array}$ & $\mathrm{p}$ value \\
\hline Increasing to obese $\mathrm{c}^{\mathrm{c}}$ & 2.22 & $1.38,3.58$ & 0.001 & 2.10 & $1.32,3.36$ & 0.002 \\
\hline Stable obese $\mathrm{c}^{\mathrm{c}}$ & 1.84 & $1.45,2.33$ & $<0.001$ & 1.72 & $1.36,2.17$ & $<0.001$ \\
\hline Increasing to severely obese ${ }^{c}$ & 3.04 & $1.49,6.20$ & 0.002 & 3.24 & $1.61,6.54$ & 0.001 \\
\hline Stable severely obese $\mathrm{e}^{\mathrm{c}}$ & 2.35 & $1.39,3.97$ & 0.002 & 2.20 & $1.32,3.36$ & 0.003 \\
\hline \multicolumn{7}{|c|}{$\begin{array}{l}\text { aModel } 1 \text { correcting only for age, gender, relevant medication use and oral contraceptive use. } \\
\text { bModel } 2 \text { correcting further for lifestyle variables (diet, physical activity, smoking behaviour). } \\
\text { c'Normative' trajectory is set as the reference category. }\end{array}$} \\
\hline
\end{tabular}

Table 4. Unstandardised regression coefficients (b), $95 \%$ confidence intervals and $p$ values for the associations between BMI-trajectory membership and fibrinogen

\begin{tabular}{|c|c|c|c|c|c|c|}
\hline & \multicolumn{3}{|c|}{ Model $1^{\mathrm{a}}$} & \multicolumn{3}{|c|}{ Model $2^{\mathrm{b}}$} \\
\hline & $\mathrm{b}$ & $\begin{array}{l}95 \% \text { confidence } \\
\text { interval }\end{array}$ & $\mathrm{p}$ value & $\mathrm{b}$ & $\begin{array}{l}95 \% \text { confidence } \\
\text { interval }\end{array}$ & $\mathrm{p}$ value \\
\hline Increasing to obese $\mathrm{e}^{\mathrm{c}}$ & 48.19 & $-19.91,116.29$ & 0.165 & 43.14 & $-25.86,112.13$ & 0.220 \\
\hline Stable obese ${ }^{c}$ & 19.83 & $-12.91,52.57$ & 0.234 & 19.85 & $-13.49,53.19$ & 0.242 \\
\hline Increasing to severely obese ${ }^{c}$ & 155.25 & $55.86,254.63$ & 0.002 & 153.42 & $53.48,253.37$ & 0.003 \\
\hline Stable severely obese $\mathrm{c}^{\mathrm{c}}$ & 68.40 & $-5.30,142.10$ & 0.069 & 69.35 & $-5.11,143.81$ & 0.068 \\
\hline
\end{tabular}

${ }^{a}$ Model 1 correcting only for age, gender, relevant medication use and oral contraceptive use.

${ }^{b}$ Model 2 correcting further for lifestyle variables (diet, physical activity, smoking behaviour).

'Normative' trajectory is set as the reference category.

mation of the BIC, BLRT, posterior probabilities and number of subjects per class are visualised in table 1.

Characteristics of the five classes are shown in table 2, including the $95 \%$ confidence intervals around the estimated BMI values of each class at each visit as demonstrated in figure 1. It can be seen that the different trajectories do not differ a great deal on lifestyle variables, although the increasing to severely obese trajectory represents the only class with no smokers. Furthermore, this class had the highest number of subjects on a restricted diet (i.e., low-salt, low-fat, low-cholesterol or vegetarian), and the number of subjects engaging in sports activities was comparable to that of subjects classified into the normative trajectory.

The results of the associations between the BMI trajectories and CRP/fibrinogen at visit 4 are shown in tables 3 and 4. In all models, the normative subgroup was used as the reference category. For CRP, all subgroups showed significantly higher values in model 1 (1.84-3.04 times higher) when compared to the normative subgroup. Correcting for relevant lifestyle variables did not affect these associations considerably. For fibrinogen, the results showed a slightly different picture. Although all subgroups displayed higher levels of fibrinogen compared to the normative subgroup (between 19.83 and $155.25 \mathrm{mg} / \mathrm{dl}$ higher in model 1), only the difference between the increasing to severely obese and the normative subgroup 
Hoekstra et al.: Effects of Long-Term Developmental Patterns of Adiposity on Levels of C-Reactive Protein and Fibrinogen among North-American Men and Women: The Spokane Heart Study

Table 5. Unstandardised regression coefficients (b), 95\% confidence intervals and p values for the associations between BMI-trajectory and change scores of CRP and fibrinogen

\begin{tabular}{|c|c|c|c|c|c|c|}
\hline & \multicolumn{3}{|l|}{ CRP } & \multicolumn{3}{|c|}{ Fibrinogen } \\
\hline & $\mathrm{b}$ & $\begin{array}{l}95 \% \text { confidence } \\
\text { interval }\end{array}$ & $\mathrm{p}$ value & $\mathrm{b}$ & $\begin{array}{l}95 \% \text { confidence } \\
\text { interval }\end{array}$ & $\mathrm{p}$ value \\
\hline \multicolumn{7}{|l|}{ Model 1} \\
\hline Increasing to obese $\mathrm{e}^{\mathrm{c}}$ & 1.41 & $0.37,2.46$ & 0.008 & 44.64 & $-69.40,158.69$ & 0.442 \\
\hline Stable obese $\mathrm{c}^{\mathrm{c}}$ & 0.26 & $-0.27,0.80$ & 0.336 & 16.83 & $-38.70,71.70$ & 0.547 \\
\hline Increasing to severely obese $\mathrm{e}^{\mathrm{c}}$ & 0.24 & $-1.32,1.80$ & 0.760 & 79.28 & $-87.08,245.64$ & 0.349 \\
\hline Stable severely obese ${ }^{c}$ & -0.22 & $-1.45,1.01$ & 0.727 & 9.98 & $-113.38,133.34$ & 0.874 \\
\hline \multicolumn{7}{|l|}{ Model 2} \\
\hline Increasing to obese ${ }^{c}$ & 1.36 & $0.32,2.41$ & 0.011 & 49.02 & $-66.57,164.60$ & 0.405 \\
\hline Stable obese ${ }^{c}$ & 0.17 & $-0.37,0.72$ & 0.532 & 17.04 & $-38.84,72.92$ & 0.549 \\
\hline Increasing to severely obese $\mathrm{e}^{\mathrm{c}}$ & 0.34 & $-1.23,0.90$ & 0.673 & 84.89 & $-82.43,252.22$ & 0.319 \\
\hline Stable severely obese ${ }^{c}$ & -0.29 & $-1.52,0.95$ & 0.650 & 10.78 & $-113.87,135.43$ & 0.865 \\
\hline
\end{tabular}

was statistically significant, and the difference between the normative and the stable severely obese subgroup was borderline significant $(p=0.069)$.

To evaluate the importance of the BMI trajectories relative to each other, the above analyses were also run with different reference categories. Interestingly, the 'unfavourable' trajectories only differed slightly from each other on both CRP and fibrinogen levels, although differences in fibrinogen levels were more profound. When comparing the stable obese and the increasing towards obesity trajectory (both showing similar BMI values at the last visit (table 2), large differences in both CRP and fibrinogen were apparent, although these differences were only borderline significant. Further comparisons are not presented here, but can be partly drawn from the results presented in tables 3 and 4.

In table 5, the same analyses are presented as in table 3 and 4 , but with the calculated change scores of CRP and fibrinogen (visit 4 minus visit 1) as the outcome variables. Again, the normative trajectory was set as the reference category. For CRP, much smaller regression coefficients appeared, and only the difference in change scores between the increasing to obese and the normative trajectory was statistically significant. This regression coefficient was, however, difficult to interpret; 1.41 can be interpreted as the difference in CRP change score between the increasing to obese trajectory and the normative trajectory, indicating that the former subgroup increased more in CRP over time (table 2). For fibrinogen, smaller regression coefficients were observed, in particular the regression coefficient comparing the change scores of the stable severely obese and the normative subgroups (table 2).

The additional analyses or the results of 'conventional' analyses are presented in tables 6 and 7. Change scores of BMI were calculated and related to CRP and fibrinogen levels at visit 4 in table 6 . This table shows that BMI change scores were positively associated with CRP and fibrinogen levels at visit 4, although regression coefficients were only statistically significant for analyses with CRP. This regression coefficient (adjusted; model 2) can be interpreted as follows: an increase in BMI change score of 1 point between visits 1 and 4 resulted in 1.11 times higher CRP levels at visit 4, whereas for fibrinogen this resulted in a by $3.65 \mathrm{mg} / \mathrm{dl}$ higher level. 
Table 6. Unstandardised regression coefficients (b), 95\% confidence intervals and $p$ values for the associations between change scores of BMI and CRP and fibrinogen levels at visit 4

\begin{tabular}{|c|c|c|c|c|c|c|}
\hline & \multicolumn{3}{|l|}{ CRP } & \multicolumn{3}{|c|}{ Fibrinogen } \\
\hline & $\mathrm{b}$ & $\begin{array}{l}95 \% \text { confidence } \\
\text { interval }\end{array}$ & $\mathrm{p}$ value & $\mathrm{b}$ & $\begin{array}{l}95 \% \text { confidence } \\
\text { interval }\end{array}$ & $\mathrm{p}$ value \\
\hline \multicolumn{7}{|l|}{ Model 1a } \\
\hline BMI change score & 1.10 & $1.06,1.15$ & $<0.001$ & 3.73 & $-1.69,9.15$ & 0.177 \\
\hline \multicolumn{7}{|l|}{ Model 2b } \\
\hline BMI change score & 1.10 & $1.06,1.15$ & $<0.001$ & 3.65 & $-1.81,9.12$ & 0.189 \\
\hline
\end{tabular}

${ }^{a}$ Model 1 correcting only for age, gender, relevant medication use and oral contraceptive use

${ }^{\mathrm{b}}$ Model 2 correcting further for lifestyle variables (diet, physical activity, smoking behaviour)

Table 7. Unstandardised regression coefficients (b), 95\% confidence intervals and p values for the associations between change scores of BMI and change scores of CRP and fibrinogen levels

\begin{tabular}{|c|c|c|c|c|c|c|}
\hline & \multicolumn{3}{|l|}{ CRP } & \multicolumn{3}{|c|}{ Fibrinogen } \\
\hline & $\mathrm{b}$ & $\begin{array}{l}95 \% \text { confidence } \\
\text { interval }\end{array}$ & $\mathrm{p}$ value & $\mathrm{b}$ & $\begin{array}{l}95 \% \text { confidence } \\
\text { interval }\end{array}$ & $\mathrm{p}$ value \\
\hline \multicolumn{7}{|l|}{ Model 1a } \\
\hline BMI change score & 0.18 & $0.09,0.27$ & $<0.001$ & 5.01 & $-3.92,13.94$ & 0.271 \\
\hline \multicolumn{7}{|l|}{ Model 2b } \\
\hline BMI change score & 0.19 & $0.10,0.27$ & $<0.001$ & 5.48 & $-3.51,14.47$ & 0.232 \\
\hline
\end{tabular}

${ }^{a}$ Model 1 correcting only for age, gender, relevant medication use and oral contraceptive use.

${ }^{b}$ Model 2 correcting further for lifestyle variables (diet, physical activity, smoking behaviour).

Small effects are also shown in table 7 , where the outcome variable is the change score of CRP/fibrinogen. An increase in the BMI change score of 1 point was associated with an increase of $0.19 \mathrm{mg} / \mathrm{dl}$ in CRP change score and $5.48 \mathrm{mg} / \mathrm{dl}$ in fibrinogen change score (again, the analyses with CRP were statistically significant and those with fibrinogen were not).

\section{Discussion}

The current study investigated the heterogeneity of BMI development over 6 years. We identified six distinct developmental trajectories of BMI. Subsequently, we assessed the associations between these trajectories and CRP and fibrinogen levels determined at visit 4 as well as change scores of these levels. 'Unfavourable' BMI trajectories differed to a great extent from the normative trajectory with regard to CRP and fibrinogen levels at visit 4, but to a much lesser extent with regard to their CRP and fibrinogen change scores. When 'conventional' analyses were conducted (i.e., replacing the BMI trajectories by BMI change scores), much smaller regression coefficients were observed. 
Hoekstra et al.: Effects of Long-Term Developmental Patterns of Adiposity on Levels of C-Reactive Protein and Fibrinogen among North-American Men and Women: The Spokane Heart Study

\section{Heterogeneity in BMI Development and Low-Grade Inflammation}

To our knowledge, this is the first population-based study that investigated the effects of distinct trajectory shapes of adiposity on markers of low-grade inflammation. There are some studies exploring the relationship between the development of adiposity and markers of lowgrade inflammation, such as CRP and fibrinogen, over time. However, although a number of studies is available, comparison with these studies is difficult for two important reasons. First, definitions of adiposity development are inconsistent across studies. For example, some researchers categorised study participants in arbitrary subgroups and pulled participants who lost weight throughout the follow-up period together with stable-weight participants [41], whereas others categorised according to established criteria [16]. In some studies, only change scores were calculated [42] when examining the development of body weight over time. Second, many terms for weight instability, such as weight variability, weight fluctuation or weight cycling, are used, but it is unclear if and how these definitions vary. Although the statistical methodology and/or definition differs between these studies, they mostly point consistently to negative health consequences, including death, when increased weight variability, weight cycling, weight fluctuation or weight increase is apparent $[42,43]$. While these studies allow for interesting and valuable results, they do not give insight in the trajectory of adiposity from the beginning to the end of follow-up and its effects on CRP or fibrinogen levels. Importance of these trajectories for traditional CVD markers $[44,45]$ and psychological health $[21,23,46]$ has been highlighted previously and should not remain unnoticed in this setting either. In our study, we found six classes of BMI development, each with a distinct trajectory. Comparable trajectories were found in previous cohorts consisting of children and older adults in particular. For example, Kuchibhatla et al. [24] analysed a community-based sample aged 65-105 years and identified a normal-weight, an overweight and an obese trajectory, but failed to detect increasing trajectories. Stable trajectories comparable to our trajectories were identified by Botoseneanu and Liang [25], Rosenberger et al. [47] and Mustillo et al. [23, 46], whereas trajectories showing more variation over time were revealed in other studies [20,23,46,48]. Generally, trajectories of similar shape are found across different cohorts, and decreasing trajectories are scarcely detected in observational studies. As found also in other studies $[11,16,49]$, the 'unfavourable' classes (i.e., increasing trajectories or stable overweight/obese trajectories) in our study showed higher levels of low-grade inflammation markers compared to the normative class. Interestingly, however, the 'unfavourable' trajectories did not differ significantly from each other. Moreover, although the BMI trajectories clearly differed with respect to their visit-1- and visit-4 values of CRP and fibrinogen, the increase in these markers differed only slightly over time; it appeared that only the two increasing trajectories (although not to the same extent) differed from the normative trajectory with regard to their CRP and fibrinogen change scores. As BMI and CRP in particular were hypothesised to be associated longitudinally, it can be concluded that stable trajectories would not necessarily have increased change scores. This can also be seen in table 2 where descriptive information by BMI trajectory is represented. These findings highlight the fact that the sole analysis of change scores can be misleading if no actual change over time is apparent in the sample, even though baseline differences are clear.

Even though we identified six distinct trajectories of body weight development, where some showed degrees of variation in trajectory, these trajectories were all still relatively stable regarding their development of BMI. Therefore, we should be aware of the fact that the lack of variation in longitudinal development in this sample may mask even larger differences in CRP or fibrinogen levels, although trajectories with greater variation are generally found in younger cohorts [23, 46, 50] and cohorts with a very long follow-up period [51].

By the classification of the participants in six distinct trajectory classes, information on the course of BMI during visits 1, 2, 3 and 4 were taken into account, which is ignored when 
Hoekstra et al.: Effects of Long-Term Developmental Patterns of Adiposity on Levels of C-Reactive Protein and Fibrinogen among North-American Men and Women: The Spokane Heart Study

analysing BMI development using change scores. Therefore, the elevated CRP values in the stable trajectories (i.e., including subjects that do not change over time) will only have been detected by this approach and would have partly been obscured, if we had performed an analysis solely with BMI change scores. However, categorisation of subjects in six distinct subgroups did cause some information loss, highlighting the importance of both approaches.

\section{Methodological Issues: Strengths and Limitations}

We used observational data collected at multiple times to investigate our research questions as thoroughly as possible. We had information on important confounders (e.g., oral contraceptive use, medication use, lifestyle factors) and were able to correct for those in our models, although we obtained information on these factors (that are known to influence lowgrade inflammation) at baseline only, but not continuously throughout the follow-up period. Further, we used up to date statistical techniques to unravel heterogeneity in adiposity development. LCGM categorise participants on the basis of their developmental pattern instead of being based on predefined subgroups [37,52]. These techniques have shown to be very promising for applications within obesity research [21-23, 46, 53, 54] and do provide researchers as well as clinicians with new insights for further research, in particular due to their data-driven nature. The 'unfavourable' trajectories that were identified with LCGM in our study are mostly based on relatively few participants $(n=8,15$ and 18). Although these are positive findings in terms of public health, small sample sizes do challenge elaborate statistical analyses. These small sizes might further complicate the generalizability of the BMI trajectories. Surprisingly, subjects classified into the increasing to severely obese trajectory $(\mathrm{n}=8)$ reported a relatively healthy lifestyle (non-smoking, high sports participation, often restricting their diets), but still increased approximately 8 BMI points over 8 years. These findings could either suggest socially desirable answering [55] or might point towards a nonrepresentative subgroup.

The importance of body fat distribution, as opposed to BMI, for the quantification of CVD risk has been highlighted in many studies $[56,57]$. Unfortunately, the Spokane Heart Study did not provide any information on body fat distribution available for the visits we used in the current study. Although some studies reported that associations between measures of fat distribution, such as waist circumference, and measures of CVD were stronger compared to associations between BMI and measures of CVD [58], others found associations of similar strength, especially for Caucasian persons [59]. Nevertheless, the utilisation of BMI as a measure of adiposity is very common in scientific research as well as within the World Health Organization (WHO), allowing for a broad comparison of the results. This is especially relevant for body weight/adiposity trajectory research, where BMI is the most common adiposity measure. It should be noted, however, that BMI does not appropriately quantify the ratio between fat mass and fat-free mass, which additionally considers other factors such as nutritional status and cardio-respiratory fitness that are known to affect cardiovascular morbidity and mortality.

\section{Conclusions and Implications}

In conclusion, the results of this study show that in a community-based sample of males and females the development of BMI is heterogeneous; six distinct trajectory shapes could be identified. Statistically significant differences in CRP and fibrinogen levels were apparent between the trajectories, where the 'unfavourable' trajectories differed from the normative trajectory and differences were more profound for CRP than for fibrinogen. Our identified BMI trajectories partly confirm previous findings from studies with varying subject characteristics, and many studies demonstrated negative health consequences in relation to 'unfavourable' trajectories $[20,23,46]$. Clinical research could, therefore, explore the clinical 
Hoekstra et al.: Effects of Long-Term Developmental Patterns of Adiposity on Levels of C-Reactive Protein and Fibrinogen among North-American Men and Women: The Spokane Heart Study

utility of these distinct trajectories, relative to each other, in disease tracking and management systems. Overall, more longitudinal research is needed to understand the short- and longterm benefits and risks associated with sustained weight loss, weight gain and weight fluctuation.

\section{Acknowledgements}

This work was partly supported by a personal travel grant awarded by the EMGO Institute for Health and Care Research and the VU University.

\section{Disclosure Statement}

There are no competing interests to declare.

\section{References}

1 Finucane MM, Stevens GA, Cowan MJ, Danaei G, Lin JK, Paciorek CJ, et al: National, regional, and global trends in body-mass index since 1980: systematic analysis of health examination surveys and epidemiological studies with 960 country-years and 9.1 million participants. Lancet 2011;377:557-567.

2 Blagojevic M, Jinks C, Jeffery A, Jordan KP: Risk factors for onset of osteoarthritis of the knee in older adults: a systematic review and meta-analysis. Osteoarthritis Cartilage 2010;18:24-33.

-3 Renehan AG, Tyson M, Egger M, Heller RF, Zwahlen M: Body-mass index and incidence of cancer: a systematic review and meta-analysis of prospective observational studies. Lancet 2008;371:569-578.

4 Young T, Skatrud J, Peppard PE: Risk factors for obstructive sleep apnea in adults. JAMA 2004;291:2013-2016.

5 Nguyen T, Lau DC: The obesity epidemic and its impact on hypertension. Can J Cardiol 2012;28:326-333.

-6 Rexrode KM, Hennekens CH, Willett WC, Colditz GA, Stampfer MJ, Rich-Edwards JW, et al: A prospective study of body mass index, weight change, and risk of stroke in women. JAMA 1997;277:1539-1545.

7 Yudkin JS, Eringa E, Stehouwer CD: 'Vasocrine' signalling from perivascular fat: a mechanism linking insulin resistance to vascular disease. Lancet 2005;365:1817-1820.

-8 Wijnstok NJ, Twisk JW, Young IS, Woodside JV, McFarlane C, McEneny J, et al: Inflammation markers are associated with cardiovascular diseases risk in adolescents: the Young Hearts Project 2000. J Adolesc Health 2010; 47:346-351.

-9 Ridker PM, Hennekens CH, Buring JE, Rifai N: C-reactive protein and other markers of inflammation in the prediction of cardiovascular disease in women. N Engl J Med 2000;342:836-843.

10 Yudkin JS, Stehouwer CD, Emeis JJ, Coppack SW: C-reactive protein in healthy subjects: associations with obesity, insulin resistance, and endothelial dysfunction: a potential role for cytokines originating from adipose tissue? Arterioscler Thromb Vasc Biol 1999;19:972-978.

11 Weyer C, Yudkin JS, Stehouwer CD, Schalkwijk CG, Pratley RE, Tataranni PA: Humoral markers of inflammation and endothelial dysfunction in relation to adiposity and in vivo insulin action in Pima Indians. Atherosclerosis 2002;161:233-242.

12 Ridker PM, Buring JE, Shih J, Matias M, Hennekens CH: Prospective study of C-reactive protein and the risk of future cardiovascular events among apparently healthy women. Circulation 1998;98:731-733.

13 Danesh J, Collins R, Appleby P, Peto R: Association of fibrinogen, C-reactive protein, albumin, or leukocyte count with coronary heart disease: meta-analyses of prospective studies. JAMA 1998;279:1477-1482.

14 Pearce MS, Ahmed A, Tennant PW, Parker L, Unwin NC: Lifecourse predictors of adult fibrinogen levels: the Newcastle Thousand Families Study. Int J Cardiol 2012;155:206-211.

15 Nazmi A, Gonzalez DC, Oliveira IO, Horta BL, Gigante DP, Victora CG: Life course weight gain and C-reactive protein levels in young adults: findings from a Brazilian birth cohort. Am J Hum Biol 2009;21:192-199.

16 Nguyen XM, Lane J, Smith BR, Nguyen NT: Changes in inflammatory biomarkers across weight classes in a representative US population: a link between obesity and inflammation. J Gastrointest Surg 2009;13:12051212.

17 Zhang H, Tamakoshi K, Yatsuya H, Murata C, Wada K, Otsuka R, et al: Long-term body weight fluctuation is associated with metabolic syndrome independent of current body mass index among Japanese men. Circ J 2005;69:13-18.

18 Tamakoshi K, Yatsuya H, Kondo T, Ishikawa M, Zhang H, Murata C, et al: Long-term body weight variability is associated with elevated C-reactive protein independent of current body mass index among Japanese men. Int J Obes Relat Metab Disord 2003;27:1059-1065. 
Hoekstra et al.: Effects of Long-Term Developmental Patterns of Adiposity on Levels of C-Reactive Protein and Fibrinogen among North-American Men and Women: The Spokane Heart Study

19

20

$>21$

22

Hoekstra T, Barbosa-Leiker C, Koppes L, Twisk J: Developmental trajectories of body mass index throughout the life course: an application of Latent Class Growth (Mixture) Modelling. Longit Life Course Stud 2011;2: 319-330.

$>23$ Mustillo S, Worthman C, Erkanli A, Keeler G, Angold A, Costello EJ: Obesity and psychiatric disorder: developmental trajectories. Pediatrics 2003;111:851-859.

-24 Kuchibhatla MN, Fillenbaum GG, Kraus WE, Cohen HJ, Blazer DG: Trajectory classes of body mass index in a representative elderly community sample. J Gerontol A Biol Sci Med Sci 2013;68:699-704.

-25 Botoseneanu A, Liang J: Latent heterogeneity in long-term trajectories of body mass index in older adults. J Aging Health 2013;25:342-363.

-26 Tuttle KR, Short RA: Longitudinal relationships among coronary artery calcification, serum phosphorus, and kidney function. Clin J Am Soc Nephrol 2009;4:1968-1973.

-27 Wright BR, Barbosa-Leiker C, Hoekstra T: Law enforcement officer versus non-law enforcement officer status as a longitudinal predictor of traditional and emerging cardiovascular risk factors. J Occup Environ Med 2011; 53:730-734.

28 Barbosa-Leiker C, Wright BR, Burns GL, Parks CD, Strand PS: Longitudinal measurement invariance of the metabolic syndrome: is the assessment of the metabolic syndrome stable over time? Ann Epidemiol 2011;21: 111-117.

29 Mielke CH, Shields JP, Broemeling LD: Coronary artery calcium scores for men and women in a large asymptomatic population. Cardiovasc Dis Prevent 1999;2:194-198.

30 Mokdad AH, Serdula MK, Dietz WH, Bowman BA, Marks JS, Koplan JP: The spread of the obesity epidemic in the United States, 1991-1998. JAMA 1999;282:1519-1522.

-31 Pearson TA, Mensah GA, Alexander RW, Anderson JL, Cannon RO 3rd, Criqui M, et al: Markers of inflammation and cardiovascular disease: application to clinical and public health practice: a statement for healthcare professionals from the Centers for Disease Control and Prevention and the American Heart Association. Circulation 2003;107:499-511.

32 Mplus Statistical Analysis with Latent Variables. User's Guide. Los Angeles, Muthén \& Muthén. www.statmodel. com/download/usersguide/Mplus\%20user\%20guide\%20Ver_7_r6_web.pdf(last accessed April 8, 2014).

33 Mplus 7.0. Los Angeles, Muthén \& Muthén.

-34 Muthén BO, Muthén LK: Integrating person-centered and variable-centered analyses: growth mixture modeling with latent trajectory classes. Alcohol Clin Exp Res 2000;24:882-891.

-35 Muthén BO: Beyond SEM: general latent variable modeling. Behaviormetrika 2002;29:81-117.

-36 Muthén BO, Shedden K: Finite mixture modeling with mixture outcomes using the EM algorithm. Biometrics 1999;55:463-469.

$>37$ Jung T, Wickrama KAS: An introduction to latent class growth analysis and growth mixture modeling. Soc Personal Psychol Compass 2008;2:302-317.

-38 Nylund KL, Asparouhov T, Muthén BO: Deciding on the number of classes in latent class analysis and growth mixture modelling?: a Monte Carlo simulation study. Struct Equat Modeling 2007;14:535-569.

39 Raftery AE: Bayesian model selection in social research. Sociol Methodol 1995;25:111-163.

-40 McLachlan G, Peel D: Finite Mixture Models. New York, Wiley \& Sons, 2000.

-41 Lloyd-Jones DM, Liu K, Colangelo LA, Yan LL, Klein L, Loria CM, et al: Consistently stable or decreased body mass index in young adulthood and longitudinal changes in metabolic syndrome components: the Coronary Artery Risk Development in Young Adults Study. Circulation 2007;115:1004-1011.

-42 Bot M, Spijkerman AM, Twisk JW, Verschuren WM: Weight change over five-year periods and number of components of the metabolic syndrome in a Dutch cohort. Eur J Epidemiol 2010;25:125-133.

$>43$ Lee JS, Kawakubo K, Kobayashi Y, Mori K, Kasihara H, Tamura M: Effects of ten year body weight variability on cardiovascular risk factors in Japanese middle-aged men and women. Int J Obes Relat Metab Disord 2001; 25:1063-1067.

-44 Ferreira I, Twisk JW, van Mechelen W, Kemper HC, Stehouwer CD: Development of fatness, fitness, and lifestyle from adolescence to the age of 36 years: determinants of the metabolic syndrome in young adults: the Amsterdam Growth and Health Longitudinal Study. Arch Intern Med 2005;165:42-48.

$>45$ Wills AK, Hardy RJ, Black S, Kuh DJ: Trajectories of overweight and body mass index in adulthood and blood pressure at age 53: the 1946 British birth cohort study. J Hypertens 2010;28:679-686.

-46 Mustillo SA, Hendrix KL, Schafer MH: Trajectories of body mass and self-concept in black and white girls: the lingering effects of stigma. J Health Soc Behav 2012;53:2-16.

$>47$ Rosenberger PH, Ning Y, Brandt C, Allore H, Haskell S: BMI trajectory groups in veterans of the Iraq and Afghanistan wars. Prev Med 2011;53:149-154.

-48 Clarke P, O’Malley PM, Jonhston LD, Schulenberg JE: Social disparities in BMI trajectories across adulthood by gender, race/ethnicity and lifetime socio-economic position: 1986-2004. Int J Epidemiol 2009;38:499-509.

$>49$ Ford ES: Body mass index, diabetes, and C-reactive protein among U.S. adults. Diabetes Care 1999;22:19711977. 
Hoekstra et al.: Effects of Long-Term Developmental Patterns of Adiposity on Levels of C-Reactive Protein and Fibrinogen among North-American Men and Women: The Spokane Heart Study

50 Garden FL, Marks GB, Simpson JM, Webb KL: Body mass index (BMI) trajectories from birth to 11.5 years: relation to early life food intake. Nutrients 2012;4:1382-1398.

51 Jun HJ, Corliss HL, Nichols LP, Pazaris MJ, Spiegelman D, Austin SB: Adult body mass index trajectories and sexual orientation: the Nurses' Health Study II. Am J Prev Med 2012;42:348-354.

52 Muthén B: The potential of growth mixture modelling. Infant Child Dev 2006;15:623-625.

-53 Ventura AK, Loken E, Birch LL: Developmental trajectories of girls' BMI across childhood and adolescence. Obesity (Silver Spring) 2009;17:2067-2074.

54 Pryor LE, Tremblay RE, Boivin M, Touchette E, Dubois L, Genolini C, et al: Developmental trajectories of body mass index in early childhood and their risk factors: an 8-year longitudinal study. Arch Pediatr Adolesc Med 2011;165:906-912.

55 Willett WC, Howe GR, Kushi LH: Adjustment for total energy intake in epidemiologic studies. Am J Clin Nutr 1997;65(4 suppl):1220S-1228S; discussion 1229S-1231S.

-56 Ferreira I, Twisk JW, van Mechelen W, Kemper HC, Seidell JC, Stehouwer CD: Current and adolescent body fatness and fat distribution: relationships with carotid intima-media thickness and large artery stiffness at the age of 36 years. J Hypertens 2004;22:145-155.

57 Ketel IJ, Volman MN, Seidell JC, Stehouwer CD, Twisk JW, Lambalk CB: Superiority of skinfold measurements and waist over waist-to-hip ratio for determination of body fat distribution in a population-based cohort of Caucasian Dutch adults. Eur J Endocrinol 2007;156:655-661.

58 Ho SC, Chen YM, Woo JL, Leung SS, Lam TH, Janus ED: Association between simple anthropometric indices and cardiovascular risk factors. Int J Obes Relat Metab Disord 2001;25:1689-1697.

59 Christian AH, Mochari H, Mosca LJ: Waist circumference, body mass index, and their association with cardiometabolic and global risk. J Cardiometab Syndr 2009;4:12-19. 